\title{
Viajes a Flandes e Inglaterra ¿Cabotaje o recta via? ${ }^{1}$
}

\author{
ANTONIO ORTEGA VILLOSLADA
}

\begin{abstract}
RESUMEN
El inicio de los viajes atlánticos de los marinos del Mediterráneo hacia Flandes e Inglaterra se produce en la segunda mitad del siglo XIII mediante los viajes directos entre Galicia y la Bretaña francesa. Sin embargo, la navegación de cabotaje no se producirá hasta la primera mitad del siglo XIV.

PALABRAS CLAVE

Mallorca, Navegación, Atlántico, Inglaterra y Flandes

ABSTRACT

The beginning of the Atlantic voyages of the marines of the Mediterranean toward Flanders and England, came about during the second half of the XIII century by means of the direct voyages between Galicia and the French Brittany. Whereas the coasting trade navigation was not standarized until the first half of the XIV century.

KEYWORDS

Majorca, Navigation, Atlantic, England and Flanders
\end{abstract}

\section{INTRODUCCIÓN}

...La navegación directa a Inglaterra desde los puertos del mediterráneo, que sin duda empezó a fines del siglo XIII, debía de ser peligrosísima tanto por los riesgos del largo viage y de la braveza de los mares británicos, estando la náutica aún muy atrasada, como por la continua pyratería de aquellos Isleños, que entonces ni sabian comerciar ni navegar, ni dexaban hacerlo pacificamente a los demás. ${ }^{2}$

En las relaciones comerciales entre los centros económicos del mediterráneo y Brujas el enlace vía marítima supuso un punto de inflexión ya que abria las puertas al comercio marítimo en gran escala de materias básicas para la industria, especialmente la pañera, que debido a su volumen y a su

\footnotetext{
El presente trabajo forma parte de la tesis doctoral que estoy realizando en la UNED, dirigida por D. José Luis Martín, bajo el título de: El Reino de Mallorca y el mundo atlántico.

${ }^{2}$ Capmany I Montpalau, A. Memorias históricas..., vol. I, p. 327.
} 
escasa rentabilidad en pequeña escala resultaban muy costosas en el transporte terrestre.

Ante el enlace marítimo entre ambos mundos, los historiadores se han tenido que preguntar necesariamente sobre la forma en que dichas mercancías eran transportadas y si los medios técnicos de la época permitían su transporte de forma eficiente. Ante esta pregunta se han formado dos grandes grupos, coincidentes con ambas posturas, sobre el tipo de navegación empleada: cabotaje o recta via.

Sottas $^{3}$ opina que los viajes se realizaban de forma directa sin escalas. De la misma forma opinan Petti Balbi ${ }^{4}$ o Touchard ${ }^{5}$. Para Roberto Sabatino López ${ }^{6}$ esta ruta directa únicamente pudo ser utilizada por las galeras, como consecuencia de su adaptación al Atlántico debido a los requerimientos comerciales, pues los veleros no tendrían capacidad para afrontar este trayecto. Más lejos va la profesora Ferreira al afirmar que las naves de la época estarían bien preparadas para soportar un viaje directo, contando únicamente con las escalas necesarias ${ }^{7}$ para no tener que navegar por la costa, alejándose así de la acción de los piratas anglo-gascones. De esta forma, el tráfico de cabotaje quedaba en manos de las poblaciones gallegas, cántabras y vascas, quienes habrían mostrado el camino a Flandes a los mediterráneos.

Otros autores apuestan por la ruta costera hacia Flandes e Inglaterra. Renouard ${ }^{8}$ se pregunta sobre el posible itinerario de estos viajes y, aún reconociendo que la flota genovesa que pone proa a Flandes desde 1298 hace el trayecto de forma directa, se inclina por el cabotaje como itinerario habitual ${ }^{9}$, con La Rochela como puerto principal de la ruta. Murino ${ }^{10}$ también cree en la ruta costera hacia Brujas, vía Burdeos, Olerón y La Rochela. Mollat du Jourdin ${ }^{11}$ es de la misma opinión de Renouard y a pesar de la evidencia de viajes directos, el gran conocimiento de la costa bretona que tenían

SOTTAS. Les messageries maritimes...p. 127.

" Considera que la ruta seria directa hacia el sur de inglaterra, evitando de esta forma el cabotaje.

... después de haber bordeado las costas españolas, desde el cabo de Finisterre hasta la altura de Saint-Mathieu, cortando de través el Golfo de Gascuña, la ruta que conducia del Mediterráneo a la Europa del noroeste desembocaba en Southampton, Londres o Brujas...,.... a fines del siglo XIV los genoveses crean. aparte de la ruta recta, una ruta de escalas desde el Mediterráneo... ... al Mar del Norte... Touchard. Commerce maritime breton..., p. 88. Esta afirmación se basa en la opinión de RENOUARD en Les hommes d'affaires..., p. 412-413.

"LOPEZ, R.S. Majorcan and Genoese...p. 1166

? Ferreira Priegue, E. Galicia en el comercio... p. 486. Curiosamente estas escalas imprescindibles no se realizarian en los grandes puertos como Lisboa o La Coruña.

${ }^{8}$ RenOuARo, Yves. Les hommes d'affaires italiens...

'Datos como la presencia de los hermanos Pessagno en La Rochela, donde se refugian con su buque de una tormenta, la presencia de una compañía lombarda en Galicia en 1280 o la fidelidad del Portulano de Pietro Vesconte del año 1311 le sirven para optar por esta via.

Murino, Mario, Andar per mare..

"Mollat du Joundin, Michel, Europa y el mar. 
los que elaboraban los portulanos le sirve como referencia para llegar a esta conclusión. También Abulafía considera la navegación de cabotaje, deduciéndolo, como los anteriores, de la exacta localización de ciudades como Bilbao y su puerto de Portugalete, con Sevilla como gran centro de intercambio donde los marinos mediterráneos transbordarian las mercancias a los marinos atlánticos quienes dispondrían de la tecnología necesaria - buques adaptados-para realizar el transporte.

Por último, algunos autores no se pronuncian o no se deciden por ninguna de estas opciones. Para Chaunu ${ }^{12}$ simplemente los buques del mediterráneo no son útiles en el Atlántico. Pistarino ${ }^{13}$ cree, cómo no, que la maestria en el arte de marear y la vasta experiencia marinera de los genoveses les hará adaptarse rápidamente al Atlántico, tal y como lo probaría la flota que en 1295 se mandó desde Aigües-Mortes a Normandía, en apoyo de Felipe IV. Desde Mallorca, Sevillano Colom no acaba de decidirse por ninguna de las dos rutas. Si bien duda de la ruta directa, la de cabotaje tendría el inconveniente de tener que afrontar el corso de algunos pueblos durante el trayecto tal y como queda reflejado en la documentación del Archivo del Reino de Mallorca ${ }^{14}$ donde se aduce al temor de los mallorquines a los castellanos y en especial a los hombres de Santander. ${ }^{15}$

Tal y como se ha visto en este somero repaso de los distintos autores, no existe una posición clara al respecto. Los autores de origen mediterráneo, embuídos quizás por la idea de un mar cerrado y bien conocido como es el Mediterráneo - cuya idea de la navegación atlántica queda perfectamente reflejada en las anteriores palabras de Capmany - no conciben que en el siglo XIII los mareantes tuviesen la capacidad de navegar por el Atlántico durante un período largo - entre una y dos semanas - sin tener más referencia que las señales del mar y el cielo. A esto hay que añadir el vértigo que les tenía que producir el Océano ignoto, lleno de monstruos y leyendas, dispuesto en cualquier momento a engullir buques y tripulantes.

Frente a esta idea mediterránea, desde el abierto Atlántico se ve de otra forma. La navegación directa entre la costa hispana y la Bretaña francesa o

Chaunu, P., La expansión europea (siglos XIII al XV).

'sistarino, Geo. La capitale del Mediterráneo: Genova nel medioevo, p. 95.

${ }^{14}$ ARM. C 1760, fol. 91 y siguientes. Actualmente estoy en fase de elaboración de un trabajo en el que se incluye tanto el estudio y transcripción de este documento como los documentos complementarios sobre este pleito realizados en Castilla.

...et specialiter timebant de hominibus de Sentender... El documento es la transcripción del pleito promovido por unos corsarios castellanos contra la escuadra mallorquina que los ha apresado dentro del puerto de Cartagena como represalia a la campaña realizada por dichos corsarios en aguas de Mallorca y contra mercaderes mallorquines en puertos tan lejanos del Estrecho -la concesión de la licencia de corsario viene dada por la necesidad de defender el Estrecho contra los musulmanes- como es Túnez, donde estos corsarios se apoderarán de dos cocas mallorquinas. La atenta lectura del largo pleito descubre que el temor de los mallorquines no iba dirigido contra los hombres y naves de Santander, sino contra estos corsarios - uno de ellos originario de Santander- que actuaban impunemente en el Mediterráneo contra todo aquel que comerciase con los musulmanes. 
el sur de Inglaterra no tiene ningún secreto para los hombres de los siglos XII y XIII. Los mareantes atlánticos disponian de los conocimientos técnicos y empíricos necesarios para realizar trayectos más o menos largos ${ }^{16}$ con las suficientes garantías como para exponer grandes cantidades de vidas y dinero en las expediciones cruzadas. ${ }^{17}$

\section{ENTRADA DE NAVES ATLÁNTICAS EN EL MEDITERRÁNEO}

\section{Viajes de cruzados:}

Los conocimientos de los marinos venían de siglos atrás. Desarrollados por los pueblos del norte europeo en sus incursiones hacia la Península Ibérica y el Mediterráneo ${ }^{18}$, en el siglo XII la ruta desde las costas de la Bretaña francesa (Saint Mathieu) hacia la zona de Gijón --prácticamente dirección sur- era más que conocida ${ }^{19}$. En $1147^{20}$ y según la crónica de Osbern los cruzados tardaron 5 días, incluyendo un fuerte temporal que les hizo temer por sus vidas, en realizar la travesía desde el finisterre bretón a la costa ovetense: ...Quarta feria vento incumbente prospero Balearicam majorem scilicet montium Pyreneorum ${ }^{21}$ capita undarum magnitude et fervore maris comperimus. Vespere autem tempestate oborta, omnes

${ }^{\circledR}$ En ningún caso se trataría de grandes trayectos como los que se realizarán varios siglos más tarde a partir del descubrimiento de América, sino en trayectos con una duración media de una semana. Por cierto, los marinos de finales de la Edad Media en adelante establecerán las llamadas "derrotas de veleros" por las cuales aprovechaban la circulación general atmosférica para establecer las rutas entre Europa y América, según la estación del año. No tenian el conocimiento científico necesario para entender el funcionamiento de los vientos oceánicos pero si el empírico suficiente para sacarle partido. Algo similar debía ocurrir con los marinos medievales con respecto a algunos de los aspectos más debatidos en la actualidad -al intentar estudiarlos desde la exactitud actual - como puede ser el de la orientación o la capacidad para ubicarse en el mar.

GaUtiER DALCHÉ se lamenta por la poca atención que tradicionalmente se ha dado a las diversas entradas de navios atlánticos en el Mediterráneo en épocas tan tempranas como el siglo XII e inicios del XIII: ...La présence de navires septentrionaux en Méditerranée entre la fin du Xle et le début du XIlle siècle paraît généralement sous-estimée. En Carte marine et Portulan..., p. 46.

: En la Crónica de Alfonso III se cuenta cómo en el año 844 Ramiro I rechaza a los Normandos en su intento de desembarcar en La Coruña: ... las naves de los normandos, a través del Océano septentrional, llegaron a la ciudad de Gijón y desde allí al lugar llamado Farum Brigantium...... los que sobrevivieron atacaron la ciudad de Sevilla y la pillaron pasando a cuchillo a muchos árabes... En el año 1097 una escuadra pirática formada con gentes de Flandes y Francia llega a Tierra Santa. En 1102 encontramos un pirata inglés con su Buza a las órdenes del rey de Jerusalén. Pirata que a su regreso a Inglaterra se parará en Santiago de Compostela. Ferreiro Alemparte, Arribada de Normandos..., p. 20.

${ }^{19}$ En las Gestas de los obispos de Hamburgo - hechas por Adam de Meissen, escolástico de Bremen y muerto hacia 1076 - se anota que la duración del paso de Prawle, no lejos de Plymouth, hasta la punta de Saint-Mathieu se tarda un dia y que la duración de un viaje de Saint-Mathieu hasta Ferrol, cerca de Santiago de Compostela, es de 3 días y 3 noches. Darsel, J. La vie maritime sur les côtes du Léon...p. 172, nota 31.

${ }^{20}$ De Expugnatione Olisiponis A. D. MCXLVII. En "Portugaliae Monumenta Historica, a saeculo octavo post Christum usque ad quintumdecimum. Scriptores, volumen I" páginas 391 a 405 . Reedición de Kraus Reprint LTD. Nendeln, Liechtenstein, 1967. En las páginas 406-407 de esta misma obra se encuentra la carta de Arnulto explicando de forma más resumida los avatares de la cruzada.

${ }^{21}$ El primero de enero de 1267 Nicolao lohanes, notario público del rey en Oviedo, valida el traslado de un privilegio plomado del rey Fernando en el cual se certifica la donación por su parte de varias iglesias y 
circumquaque dispersi sumus. Noctis supra modum tenebrositas atque insueta maris reumata nautas et audacissimos desperare cogebat. Auditae sunt interim syrenes horribilis sonitus, prius cum luctu, postea cum risu et cachynno, quasi insultantium castrorum clamoribus. Per totam igitur dominicae ascensionis noctem laborantibus, consors atque custos divina misericordia affuit, ut castigando castigaret et morti non traderet......Postera igitur die paululum, sedata tempestate, in Hyspania apud portum Sancti Saluatoris quae dicitur Mala-rupis feliciter applicauimus...

Arnulfo $^{22}$ en su epistola al obispo Milón, comenta que fueron en total 7 los días de travesía con una flota de unas doscientas naves: ... In VI feria ante rogationes navigavimus cum ducentis fere nauibus, et continuos septem dies et noctes in alto mari laborantes, in vigilia Ascensionis et in ipsa die solemni saevissima tempestate quassati, octauo demum die in portum Hispaniae qui Gollim dicitur cum quinquaginta fere nauibus, ceteris circumquaque dispersis...

El tercer relato sobre la cruzada de 1147 se debe a Duodechino ${ }^{23}$ de Lahnstein: ...Anno dominicae incarnationis MCXLVII, in octaua paschae, quae fuit $V$ kalendas Maii, movit navalis exercitus a Colonia. XIV kalendas lunii, venimus in portum Angliae qui Derthmute dicitur, ubi comitem A. de Areschoth cum CC fere nauibus tam Anglorum quam Flandrensium invenimus. Ibi per triduum commorati, VI feria ante rogationes navigauimus continuos octo dies et noctis in alto mari laborantes. In vigilia et sancta sollempnitate ascensionis saevissima tempestate quassati, octaua demum die in portum Hispaniae qui Gozzim dicitur cum L fere nauibus...

En $1189^{24}$ la expedición de cruzados renanos, flamencos, frisones y daneses, que acabarán conquistando Silves, después de 9 días de navegación llegan a Gozón. En $1197^{25}$ una nueva expedición a Tierra Santa promovida por el emperador Enrique VI reúne 44 naves que en su camino hacia Jerusalén siguen la ruta habitual: ... Exercitus autem imperatoris, qui de Alemania et ceteris terris eius iter lerosolimitanam per mare susceperat, transsitum fecit in Normanniam et Angliam et sic rectum cursum tenens usque in Hispaniam, eripuit civitatem Silvie de manu paganorum...

propiedades al obispo de Oviedo. El traslado incluye un documento de inicios del siglo IX en el que se hace referencia a los Pirineos de Castilla: ...et a flumine Deua usque in Transmera et per llitus maris pascua in omnibus locis sine montactico. Foris Pirineos montes, in Castella, in territorio de Maia pernominata uillam in Pontes diuisa integra per homnes suos terminos.. Rodriguez Diaz, Elena E. El libro de la "Regla Colorada» de la Catedral de Oviedo. Estudio y edición. Documento número 12, p. 332. Los cruzados estaban viendo la Cordillera Cantábrica y por la zona a la que llegaron se trataría de los montes Urriales, al sur de Llanes.

${ }_{22}$ Portugaliae Monumenta Historica... Escriptores, volumen I, p. 406.

2. Ferreiro Alemparte, J. Arribada de Normandos y cruzados..., p. 235

${ }^{24}$ Sobre las distintas ediciones de la Narratio de itinere navali peregrinorum Hierosolyman tendentium et Silviam capientium, ver FeRREIRO ALEMPARTE, J. Arribada..., p. 80

${ }^{25}$ Ferreiro Alemparte, J. Arribada de Normandos y cruzados..., p. 81. 
En $1217^{26}$ el relato anónimo recogido en la crónica de Emón, abad del monasterio premostratense holandés de Floridus Hortus, nos describe un nuevo viaje a Tierra Santa después de haber reunido una gran flota renanoflamenca de 212 buques de todo tipo en Darthmouth desde donde se dirigen a la punta de Bretaña y de allí directamente hacia Galicia, con el puerto de La Coruña como objetivo. En esta ocasión la duración del trayecto fue de 5 días con sus noches ${ }^{27}$. ... Nos igitur sequenti die cum Comite Hollandie Britanniam aduertentes, quarta feria portum Sancti Mathei attigimus, ubi secundo perlectis constitutionibus, totus eisdem legibus seruandis se astrinxit exercitus. Sequenti vero die dominico mutatis custodiis Comiti Hollandie preducatus et marescallo coloniensi postducatus committitur. Portum Sancti Mathei linquentes aquiloni vela laxauimus, qui Ulixibonam tendentibus cum omnibus suis collateralibus est utilis. Ventis itaque prosperis sed nimis lenibus usi, sexta feria proxima Phare pervenimus, quod est oppidum Galicie diues admodum. Portum habens flexuosum, turre sublimi presignata a lulio Cesare constructa...

Como se ha visto, las crónicas nos han dejado constancia de una serie de grandes expediciones militares hacia Tierra Santa, en el marco de las Cruzadas, en las que se ponía en juego gran cantidad de recursos económi$\cos$ y humanos de alto valor. Recursos que no podian ser expuestos a la aventura de unos viajes desconocidos, pues tenían que ser guardados de forma razonable, lo que implicaba el asegurar las naves y sus bienes - dentro de lo posible - utilizando rutas bien conocidas por los mareantes atlánticos. Todos estos relatos tienen como denominador común la total ausencia de referencias a puertos del Golfo de Vizcaya. En todos se indica la zona de Oviedo como lugar de arribada de las distintas flotas ${ }^{28}$, salvo en la crónica de 1217 en la que es el puerto de La Coruña el lugar de arribada después de haber avistado la costa a la altura del Cabo Ortegal.

\footnotetext{
${ }^{26}$ Relato del cual FERREIRO Alemparte hace un estudio pormenorizado en: Arribada de Normandos $y$ cruzados.

2: En la Gesta Hammburgensis ecclesiae Pontificum, de Adam, canónigo de Bremen, ha llegado hasta nosotros un itinerario marítimo -tal y como se ha comentado ya en la nota n. ${ }^{0}$ 19- con origen en el cabo Prawle -entre Dartmouth y Plymouth-y final en San Juan de Acre. Calculaba que, siempre con vientos favorables, la duración total del trayecto era de 33 dias, dividiéndolo en etapas: Del cabo Prawle a Saint Mathieu (finisterre bretón) 1 día; de Saint Mathieu a Faro (La Coruña) 3 dias con sus noches; de allí a Lisboa eran 2 dias con sus noches; de Lisboa al Estrecho 3 dias con sus noches; del Estrecho a Tarragona 4 dias con sus noches; De Tarragona a Barcelona 1 dia; De Barcelona a Marsella 1 día y 1 noche; De Marsella a Mesina - Sicilia - eran 4 días con sus noches; de Mesina a Acre 14 dias con sus noches. Recogido por FerReiro Alemparte, J. Arribadas de normandos y cruzados... p. 114-115.

${ }^{28}$ En la crónica de Osbern se hace referencia a La Coruña como zona fronteriza entre la región meridional y la occidental. Zona que es la primera que se ve al llegar recta via desde la Bretaña trancesa:

... Asi pues muy poco despues, calmada la tempestad, atracamos felizmente en Hyspania en el puerto de San Salvador que se dice Mala-rupis... ... navegando llegamos a la ribera de Ovies (Ribadavia), la cual limita con la provincia lucense. Pero dista de la ciudad de Lugo 20 millas. Navegando de nuevo llegamos a Ortigiam (cabo Ortegal). Desde aquel lugar a la torre del Faro (La Coruña) que antiguamente Julio Cesar construyó......además está situada ciertamente entre la región meridional y la occidental que es la primera tierra a la que se llega viniendo rectamente desde Bretaña... Portugaliae Monumenta..
} 


\section{Otros viajes}

Hemos visto cómo las distintas flotas de cruzados que desde el siglo XII transitan por el Mediterráneo en su ruta hacia San Juan de Acre recalan en distintos puertos del Mediterráneo, como es el caso de la cruzada de 1217 que incluso pasa el invierno en Roma, en su camino hacia Tierra Santa. Parece fuera de toda duda que la comunicación entre los patrones de las embarcaciones mediterráneas y atlánticas tuvo que producirse, a lo largo de los diversos puertos mediterráneos, tanto para hablar de las distintas rutas como de las características de sus embarcaciones.

Es bien sabido que los naocheros se especializaban en algunas rutas y fuera de ellas carecian de la información suficiente como para poder navegar con seguridad, tal y como se nos atestigua en la narración de la expedición de 1217. Después de una fuerte tormenta, algunos buques de los cruzados atlánticos, gracias a un cautivo sarraceno, llegan probablemente a la isla de Cabrera donde se refugian del temporal. Allí encontrarán algunos mercaderes que también se habian refugiado y con su ayuda podrán llegar a Mesina: ... Entretanto, los de Neuss, gracias a la intercesión e intervención de su patrono San Quirino y de otros santos, conducidos por un sarraceno que habian retenido cautivo, arribaron felizmente el sábado 13 de abril a una isla cerca de Menorca llamada Kiporoure - iCabrera?-. En este mismo puerto habian buscado refugio algunas naves de mercaderes cristianos, los cuales el 15 de abril, domingo de Pascua, comulgaron en compañía de los cruzados. $Y$ el lunes 16 de abril, recibidos algunos mercaderes en nuestras naves como guías, pusimos alegres rumbo a Messina... ${ }^{29}$

En el siglo XIII continúan las entradas de buques atlánticos en el Mediterráneo tanto a título personal como colectivo. En 1238 Thibaud, rey de Navarra, reúne en Bayona la flota con la que tiene que ir como cruzado a Tierra Santa ${ }^{30}$. Entre los años 1243 y 1245 naves cántabras participan en la ocupación del reino de Murcia bajo las órdenes de Ruy García de Santander ${ }^{31}$. Durante la estancia de San Luis como cruzado en Tierra Santa llega a su campamento, tras cruzar el Estrecho de Gibraltar, un buque construido en Noruega: ...tandis que le Roy fermoit Cezaire vint en l'ost monseigneur Alenars de Senaingan, qui nous conta que il avoit fet la nef ou réaume de Nozoe ${ }^{32}$, qui est en la fin du monde deers Occident, e le couvint passer par les destroiz de Marroch: en grant peril passa avant qu'il venist à nous... ${ }^{33}$ En 1270 San Luis inicia su segunda y fatal cruzada partiendo de

Ferreiro Alemparte. Arribada de Normandos..., p. 214

Histoire General de Bayonne, p. 284.

- Tal y como reconocía Alfonso $X$ en 1260: ...por los muchos seruicios que nos fizo et sennaladamentre por el seruicio que no fizo sobre mar en la nuestra conquista quando ganamos el regno de Murcia.... TORRES Fontes, J. La Orden de Santa Maria de España, p. 76.

Noruega

Jehan, sire de JOINVILLE. Histoire de Saint Louis. Imprerie Royale. Paris, MDCCXI. p. 103 
Aigües-Mortes en dirección a Túnez con una muy variada flota compuesta por navíos provenientes de Normandía, Bretaña, Santoña, Cataluña, Marsella, Génova y Pisa. ${ }^{34}$

Unos años más tarde -1274-, Alfonso $X$ organiza una flota para transportar todo lo necesario para su estancia en Beaucaire, donde mantendrá infructuosas entrevistas con Gregorio X con motivo de su reclamación por el fecho del Imperio. Ordena que naves castellanas de Galicia, Asturias y Sevilla vayan a Marsella: ... Otrosy mando cargar en Seuilla e en los puertos de Gallizia e de Asturias muchas naues de trigo e de çeuada e de vinos et de otras viandas, las que entendió que podian yr por mar... ${ }^{35}$

\section{Navegación hacia el Atlántico}

Ahora bien, ¿conocían los marinos del Mediterráneo estas rutas?, ¿estaban capacitados tanto ellos como sus naves para atrontar estas nuevas rutas? Veamos lo que nos dicen las fuentes.

Las primeras referencias que tenemos de navegación de marinos mediterráneos por aguas atlánticas en dirección Inglaterra son de abril de 1277 cuando una de las galeras de Dugus Spinola zarpa hacia Flandes desde Génova ${ }^{36}$. Lo que nos indica un buen conocimiento de la ruta marítima y, sobre todo, una perfecta adecuación de los buques a las características atlánticas. Adecuación que habria tenido lugar gracias a los conocimientos previos que habrían adquirido en puertos de intercambio como Sevilla ${ }^{37}$ y a través de la observación y conocimiento, tal y como hemos visto en las páginas anteriores, de los buques atlánticos que entraban en el Mediterráneo -incluidas las galeras atlánticas, de donde

${ }^{34}$ Según la lista de los caballeros del rey, BASTARD DE PERÉ cita como buques del Mediterráneo a las naves, buzas, buza-naves, xalandros, galeras, taridas, saetias, leños y uxiers. Mientras que los buques que llegan desde el Atlántico son naves (nefs), cocas y galeras.

${ }^{35}$ Crónica de Alfonso $X$, capítulo LIX.

${ }^{36}$ Unos años antes -1272- anunciaba Ramón Llull la necesidad de estos viajes que unian, vía maritima, puertos tan lejanos como los del Mediterráneo (desde Focea hasta Mallorca) y Brujas: ... Nos veem que moltes de mercaderíes son en que hom no pot guaanyar si doncs hom no la va cercar e portar en longues terres e en loncs viatges.. Llibre de Contemplació en Deu, capitulo 116: Com hom se pren guarda de so que fan los mercaders. Pues ya se sabe que la invención de la marina es fruto del deseo comercial de los mercaderes: Ramon, qui ymagina enans nau. mariner o mercader? Lo primer mariner fo savi mercader qui desirà anar per la mar per ço que diners pogués guanyar; per que esta mercaderia, causa de marineria. Arbre de Ciencia. Del Arbre Questional, capítulo $V:$ De les questions de les fulles del Arbre humanal / De les questions del habits, punto f: De les questions de marineria.

${ }^{37}$ En el repartimiento de Sevilla se hace mención a un total de 21 cómitres con procedencias diversas. Si bien la mayoria de ellos eran de origen italiano y catalán, algunos de ellos tenían un origen atlántico: Burdeos o Laredo. GonzÁlez, J. Repartimiento de Sevilla, tomo I, páginas 297 y 315 . También en San Juan de Acre, lugar de llegada de las expediciones, habrían tenido, anteriormente, lugar el intercambio de experiencias y conocimientos entre los mareantes de ambos mares, aparte de otras situaciones como la de los pilotos mediterráneos embarcados en las naves atlánticas como guías. 
habrian tomado el diseño-. Galeras que, por otra parte, tenían una larga experiencia en el Atlántico con Bayona ${ }^{38}$ a la cabeza suministrando a lo largo del siglo XIII galeras al monarca inglés para sus continuas guerras con Francia. En 1216 Juan sin Tierra pide a Bayona que le envíe galeras para la guerra. En 1277 -año de la primera navegación mediterránea, conocida, hacia Inglaterra- Eduardo II confirma la paz entre los Cinco Puertos y Bayona y la galera aparece, al igual que en los textos mediterráneos, como uno de los tres principales tipos de buques atlánticos: ...Le Mestre de la Neef ou de la Galère ou du vessel ou celuy soit qi la playe ou le tort avera resceu al poer qil e ses compagnons de meisme la neef ou de la Galère ou de vessel de quant que soient de ambedeux les parties puissent... ${ }^{39}$

Roberto Sabatino López estudió el porte de estas galeras y llega a la conclusión de que tenían más capacidad de carga que las naves redondas ${ }^{40}$. También son dos galeras los primeros buques en los que aparecen mallorquines en Inglaterra. En agosto de 1281 la galera, de propiedad genovesa, del mallorquín Guillem de Bone carga 267 sacas y 5 balas de lana. Mientras que en 1304 la galera del mallorquín Pere Berge carga 328 sacas y 37 balas de lana perteneciente a Lorenzo de Génova y otros italianos. ${ }^{41}$

Hay que esperar hasta 1312 para encontrar el primer buque de los llamados redondos - en concreto se trata de una coca que transporta mercancías desde Mallorca a Flandes- $-{ }^{42}$ en los viajes hacia el Norte de Europa.

${ }^{38}$ En junio de 1260 está registrada la presencia de un inglés - Pedro Arnalt de Londres-en la sevillana calle de Bayona. GonzÁlez, J. Repartimiento de Sevilla, tomo I, p. 315.

${ }^{3 y}$ GOYHENECHE, E. Bayonne et la région..., p. 533 ; documento n. ${ }^{9}$ LVII. Nótese que la enumeración de los distintos tipos de buques es idéntica a los enunciados dados en el Mediterráneo, salvo el cambio lógico del nombre del buque. Asi pues la expresión: ... la neef ou de la Galère ou de vessel..., es igual a la muy corriente expresión mediterránea: tota nau o galera o leny... De esta forma, el vessel atlántico sería el equivalente al leny mediterráneo.

${ }^{40}$... La creencia extendida de que las galeras mediterráneas eran más pequeñas que las cocas redondas del Mar del Norte no se sustenta con hechos. Las listas detalladas de los impuestos que pagan las mercancias en los puertos ingleses a finales del siglo XIII e inicios del siglo XIV claramente muestra que la carga de una galera es más grande que la de las cocas más grandes. Lopez, $R$. S. Majorcans and genoese... p. 1166, nota $n .{ }^{\ominus} 1$. No podemos olvidar que las primeras mercancias transportadas en estas galeras parecen ser más propias de un trasporte en buque redondo que no en las, supuestamente, más débiles galeras. El alumbre se constituye como el elemento básico de la exportación hacia Flandes, mientras que de vuelta las galeras se cargan principalmente con balas de lana para la industria pañera de las repúblicas italianas, especialmente para Milán.

${ }^{41}$ LOPEZ, R. S. Majorcans... 1172

${ }^{42}$... actum fuisse inter me et ipsum Petrum in instrumento naulizacionis sive locationis quam ipse mihi fecit de quadam cocha sua pro deferendo pro me et meo nomine cantaria mille sexcenta de rebus et mercibus de maionici - maioricis - ad partes Bruges... DoEHARo. Les galères..., p. 60. Documento número XXVI. 
En la fachada atlántica existía, al menos desde el siglo XII, la gran ruta de cabotaje encargada de distribuir los productos de la zona atlántica ${ }^{43}$ y que se encontraba en mano de los diversos pueblos de la zona, siendo Bayona el eje central de los intercambios entre la costa peninsular y el Norte europeo. En los estatutos de la Societas Navium Baionensium - creada entre 1207 y 1213 - se contemplan los costes de los fletes según el destino y se observa que, por el norte, llegaban hasta Flandes, mientras que por la costa norte peninsular llegaban hasta Ferrol. Siendo su principal ruta comercial la que tiene como punto de destino Flandes empleando como escalas los puertos de La Rochela y Burdeos. Pero no podemos olvidar el papel jugado en esta ruta por los marinos portugueses ${ }^{44}$, gallegos ${ }^{45}$ y de la costa norte peninsular. ${ }^{46}$

: Ruta muy frecuentada ya en época de Alfonso X el Sabio quien la empleó para ensalzar la gloria de la Virgen Maria en una de sus cantigas - la número 267 -

\author{
Entre Doir'e Mynn'en Portugal morava \\ un mercader (rico) muito que amava. \\ ...Assi toi que el ssa nav'ouve fretada \\ pera yr a Frandes; e essa vegada. \\ pois que ouve ben sa fazenda guisada, \\ foi-sse con quant'aver avia mercado \\ Mais pela costeira do gran mar d'Espanna \\ ind'aquela nave con mui gran companna.. \\ ... Levantou sas ondas fortes feramente \\ sobr'aquela nave que aquela gente..
}

\begin{abstract}
${ }^{44}$ Mercaderes portugueses se encuentran en la zona mediterránea - Marsella y Montpellier- en 1189. En Brujas a partir de 1184 y en Inglaterra en 1204 cuando Juan sin Tierra les autoriza a instalarse en la isla. En 1253 Alfonso de Portugal prohibia a los mercaderes extranjeros exportar mercancias sin importar otras de igual valor y en la relación de productos aparecen escarlatas flamencas e inglesas, paños de Gante, Brujas, Iprés, Lilie, Arrás, Tournai, Saint Omer... Al año siguiente el monarca ordenaba que todos los navios que llegaran de Francia, La Rochela u otras partes, a la ribera del Duero deberian descargar la mitad en el puerto de Gaia y la otra mitad en Porto (en 1282 el rey Denis y el obispo de Porto renegociarán este acuerdo a favor de Porto). Años más tarde —en 1293-, en la misma época de la formación de la hermandad de las Cinco Villas, formarán una comuna para el comercio con el norte europeo - Flandes, Inglaterra, Normandia, Bretaña y La Rochela - con unos fondos de al menos 100 marcos de plata en la caja de Flandes y el resto en Portugal para hacer frente a posibles pleitos o negocios. En 1297 el Concejo de la ciudad de Lisboa llegaba a un acuerdo amistoso con los patrones de los buques extranjeros debido a la re yerta habida entre ellos y los patrones lisboetas. Los portugueses forman parte del grupo de extranjeros a los que Eduardo I concede la famosa Carta Mercatoria.

Ver estos y otros puntos en Descobrimentos Portugueses.

*. Durante la segunda mitad del siglo XII las principales villas costeras -Padrón, Burgo de Faro. Noya y Pontevedra- están en manos del arzobispo de Santiago y sólo a inicios del siglo XIII fructificarán Bayona de Miño -en 1201 - y La Coruña - en 1208- como puertos de realengo. Pero no es hasta 1275 que se tienen las primeras noticias de buques de Pontevedra, Vivero, Ribadeo y La Coruña en aguas británicas y flamencas. Ver FerReirA Priegue, Elisa. Galicia en el comercio maritimo medieval.

4: En la costa cántabra, Castilla no parece dar importancia a sus puertos y sólo a finales del sigo XII se produce el interés castellano por la zona y la conquista de la costa guipuzcoana. Son los años de conce. sión de fuero a puertos como Santander - 1187-, Laredo - 1200-, Santillana del Mar -1209-o San Vicente de la Barquera -1210-. Después de la conquista del litoral guipuzcoano, rápidamente Alfonso VIII confirma el fuero de San Sebastián dado por Sancho el Sabio hacia 1180 y lo extiende a Motrico, Guetaria y Fuenterrabia. Este interes del monarca de Castilla vendrá dado por la necesidad de contar con unos puertos capaces de dar salida a la exportación de las lanas castellanas.
\end{abstract}


Ante tal concurrencia de marinas e intereses, los marinos mediterráneos tenían escasas posibilidades de encontrar su nicho comercial en esta ruta de cabotaje y únicamente una alta especialización permitiria incorporarse al flujo comercial. Si bien la especialización se lograba en la ruta directa al poner en comunicación los centros laneros del norte y sur de Europa, el cabotaje mediterráneo podrá servirse de este mismo flujo comercial y aprovechar la ruta para transportar mercancías entre los diversos puertos intermedios y poder rentabilizar el trayecto.

Hasta la fecha se ha creído que los marinos mediterráneos no pudieron entrar en el circuito del cabotaje atlántico hasta finales del siglo XIV. Fecha en la que Renouard ${ }^{47}$ encuentra a unos mercaderes italianos en La Rochela y considera que habrían sido los italianos los primeros en establecer la ruta de cabotaje entre el Mediterráneo y el mar del Norte. Sin embargo esta ruta estaba siendo utilizada por los marinos de la Corona de Aragón con, al menos, 50 años de anticipación a la fecha propuesta por Renouard.

Efectivamente, en julio de 1331 Alfonso IV ${ }^{48}$ da -ante la guerra que mantiene, junto a Jaime III de Mallorca, contra Génova - unas ordenanzas sobre normas de seguridad marítima en las que detalla las zonas de navegación y el armamento que deben llevar los distintos tipos de buques. En ellas se especifica que las cocas de menos de 2000 salmas pueden navegar hasta Sevilla y, por la costa, llegar hasta Flandes, pero no pueden pasar de Colliure o las Baleares hacia el este: ... Les coches, empero, de II milia salmes enjus puguen nauegar entro a Mayorcha, entro a Xibilia e, per tota la plage, fins en fflandres; entro a copliure en estes mars per tota la Ribera, axi com dauall se conten, e no degen passar les ylles de Mallorcha o de Menorcha ${ }^{49} .$. Vemos pues que la ruta de cabotaje hasta Flandes ya era empleada de manera regular por los súbditos de la Corona de Aragón en el primer tercio del siglo XIV.

El 6 de febrero de 1333 se acordaba una imposición sobre todo el comercio del Reino de Valencia ${ }^{50}$ con motivo de sufragar los gastos producidos por las galeras que se tenían que enviar al Estrecho y aunque la redacción no es tan clara, vuelve a hablarnos de la navegación de buques - de distintos tipos y tamaños- mediterráneos más allá del Cabo de San Vicente en dirección al Norte de Europa: ...tota Nau o Cocha, Galea, Terida o leny de $M$ quintars a ensus tro en II mille que vaia o uinga de qual se uol parts del Cap de Sent Vicent enllá, pach per lo buch LXXX solidos.

\footnotetext{
${ }^{47}$ RenOuaro, Y. Les hommes d'affaires italiens.

"MADURELl MARIMON transcribió estas ordenanzas - ver bibliografía- pero usó una segunda redacción dada en Tortosa el 15 de octubre en la que no sólo ratificaba esta primera redacción - en los folios $54 \mathrm{v}-59 \mathrm{r}$ del mismo registro $\mathrm{n} .{ }^{\circ} 537$ - sino que se ampliaban con nuevas normas de policia.

${ }^{24}$ A.C.A. Cancillería, registro $n^{\circ} 537$, fol. 48 r. La primera redacción de estas ordenanzas -abarcan los folios $45 \mathrm{r}$ a $50 \mathrm{r}$ - se da a principios de julio en Barcelona. Éstas están mejor esquematizadas en el propio documento mientras que en los folios $54 \mathrm{v}-59 \mathrm{r}$ están escritas seguidas y sin diferenciar.

A.C.A. Cancillería, registro ${ }^{\circ} .^{\circ} 537$, folios $126 \mathrm{v}-127 \mathrm{v}$
} 


\section{MEDIOS TÉCNICOS}

\section{Buques y marinos}

Si nos atenemos a la capacidad de navegación de los buques de la época y la velocidad que eran capaces de desarrollar, vemos que resulta prácticamente imposible poder realizar por la costa los trayectos dados por las distintas crónicas pues no podian navegar con la velocidad suficiente como para poderlo completar en el plazo de una semana. Ya hemos mencionado que el rumbo a seguir desde la Bretaña francesa, es decir, desde Saint-Mathieu, a la zona de Oviedo es prácticamente sur. La distancia aproximada entre ambos puntos es de algo más de 500 kilómetros, mientras que si tomamos el puerto de La Coruña como lugar de arribada esta distancia llega a los 600. También hemos visto que en 1147, con tormenta incluida, tardaron 7 u 8 días - según sea el redactor de la crónica- mientras que en la expedición de 1217 el trayecto, más largo hasta La Coruña, se realizó en 5 días. Si tomamos la duración de este último viaje vemos que las naves habrían recorrido un promedio de $100 \mathrm{ki}$ lómetros por jornada, dándonos una velocidad media de unos 5 kilómetros / hora. Lo que nos sitúa dentro de las velocidades medias que podian desarrollar los buques a plena carga.

Sin embargo si hubiesen querido ir hasta La Coruña navegando de cabotaje la distancia entre Saint-Mathieu y La Coruña se amplía hasta algo más de 1200 kilómetros, es decir más del doble de distancia, lo que les habría llevado a desarrollar unas velocidades medias que no estaban al alcance de las naves de la época. ${ }^{51}$

En el mundo atlántico musulmán, a mediados del siglo XII, se consideraba como una etapa normal - yaum- el recorrer al día entre 100 y 150 kilómetros, según los vientos ${ }^{52}$. Para los buques cristianos vemos que mantienen los mismos promedios.

También se mantienen estos promedios en el Mediterráneo. Veamos algunos ejemplos. La flota cruzada de 1217 llegará finalmente a San Juan de Acre el 26 de abril después de siete días de navegación desde Creta. La distancia entre Creta y San Juan de Acre es de unos 850 kilómetros, lo que nos

"Los estudios con las reconstrucciones de las cocas de Bremen y Kamper han dado como resultado una velocidad media máxima de 6 nudos, es decir, algo menos de 11 kilómetros/hora, con vientos de fuerza 6. Con vientos de fuerza 3 navegan a unos 3'5 nudos; con fuerza 4 a unos 4 nudos y con fuerza 5 a algo más de 5 nudos. Die Kogge. Sternstunde..., p. 26.

: PICARD. L'Ocean atlantique..., p. 189 a 193. Estudia las distancias recorridas por los buques musulmanes en aguas atlánticas según autores como AL-ZUHRI, AL-IDR_SI, IBN SAID, AL-BAKRI O AL-TADILI y llega a la conclusión de que las distancias medias recorridas oscilan entre los 90 y los 120 kilómetros, dependiendo de las circunstancias metereológicas, llegando en contadas ocasiones y como hechos excepcionales a los 150 kilómetros. 
da un promedio de 120 kilómetros/día, es decir, 5 kilómetros/hora ${ }^{53}$. Algo similar sucede con la flota cristiana que el 5 de septiembre de 1229 pone rumbo desde Salou y Tarragona hacia Mallorca. El día 7 fondean reunidos en La Palomera después de dos días de navegación, temporal incluido. Dado que la distancia es de unos 180 kilómetros, habrían mantenido una velocidad media de casi 4 kilómetros/hora.

Lo mismo ocurre con los buques dedicados al comercio. Son numerosos los documentos referentes a viajes entre Cerdeña y Mallorca en los que se tarda, según condiciones metereológicas, entre 3 y $5^{54}$ días en la travesía. Dado que hay unos 420 kilómetros entre ambas islas -apenas $100 \mathrm{kms}$. menos que desde Saint-Mathieu a Gijón - volvemos a encontrarnos con los promedios ya conocidos.

Pero no todo se debía al buque en sí, sino que los conocimientos náuticos de los marinos - tal y como nos relata Ramón Llull hacia 1272 eran los que hacian posible estas singladuras: ...Los mariners que van sobre el mar, senyer, veem que saben aesmar lo viatge quants milles ha, $e$ saben aesmar la corsa que fan lo dia e la nit, e en coneixença d'aquells vents que los son bons e qui los son contraris... ${ }^{55} \mathrm{Y}$ no sólo conocian los medios para una navegación segura, sino que también podían, en caso de tiempo contrario, calcular su posición y controlar el buque - dentro de sus escasas posibilidades - con el fin de llegar a buen puerto: ...Los savis mariners veem, Senyer, que com an vent a lur contrari, ques tenen aitant com poden e que fan voltes sà e là per la mar, e que esperen lo vent qui es bo en lur viatge ${ }^{56}$...

Hemos visto al inicio que muchos autores niegan la posibilidad de que se pudiera navegar alejado de la costa y aducen que no era lo mismo navegar en el Mediterráneo que en el Atlántico ${ }^{57}$. Parecen olvidar que las distancias en el Mare Nostrum no permiten navegar constantemente viendo la costa y que un viaje tan sencillo como es la travesía hasta Mallorca requería que al

53. . post solis occasum portum linquentes, ventis et mari plus quam pro voto blandientibus, die VIIO. sequenti die post Marci Euangeliste, portum Aconensem gaudentes intrauimus... FERrEIRO ALEMPARTE. Arribadas de..., p. 223.

${ }^{54} \mathrm{Al}$-IDRISI, al hablar de las Baleares, sitúa a Menorca al oriente de Mallorca, y en frente de Barcelona, de la que está a una jornada de distancia. Desde Menorca a Cerdeña dice que hay cuatro jornadas de navegación

\$5. Libre de Contemplació, capítulo 117: Com hom pren guarda de ço que fan los mariners.

st Libre de Contemplació en Deu, capitulo 117.

Es conocido por todos los navegantes que hayan trecuentado ambos mares que la ola del Mediterráneo es corta y rápida, frente a la más tranquila del Atlántico. Con estas caracteristicas, los temporales del Mediterráneo son - a igual fuerza de mar- dificiles de navegar, tal y como comprobarían, por ejemplo, los cruzados que en 1217 viajaban hacia Palestina: ... el 15 de agosto, dia de la Asunción, asumidos en las alas de céfiro, no tuvimos la sensación de navegar, sino de volar. hasta el punto de que los que estaban acostumbrados al mar-atlántico- y a sus trabajos, juraban no haberse visto nunca con tan airados elementos ni arrebatados por tan horrendos temporales.. Arribada de Normandos... p. 101 
menos durante prácticamente un día se navegase sin ningún tipo de referencia visual ${ }^{58}$. Lo mismo ocurre, como acabamos de ver, en la ruta entre Mallorca y Cerdeña en la que habian de pasar varios días -y sus correspondientes noches - navegando rumbo este sin ningún tipo de referencias geográficas, siendo el sol y las estrellas las únicas referencias astronómicas. Esta navegación se realiza, tal y como nos confirma Llull en el texto anterior, a la estima - e saben aesmar la corsa que fan lo dia e la nitsegún sus conocimientos técnicos y empíricos. Conocimientos que hay que tener en cuenta y que también nos describe el beato: ... Lo mariner consira galera e nau e barca, e consira vela e arbre, timo e nauxer e altres que a nau se pertanyen; $e$ aprés consira temps de navegar, els ports a los quals ha refugi, e l'stela e l'agulla e la caramida, e els vents e les milles e les corses d'aquells, e altres coses que se pertanyen a la sua art. ${ }^{59}$

También Alfonso el Sabio recoge el conocimiento que deben tener los pilotos de las naves - naocheros-:... Naocheros son llamados aquellos por cuyo seso se guian los nauios por la mar... ...que ayan en si estas quatro cosas. La una que sean sabidores de conoscer todo el techo de la mar, en quales logares, es quedo, o en qual es corriente, e que conozcan los vientos, e el cambiamiento de los tiempos, e sepan toda la otra marineria. Otrosi, deven saber las yslas, e los puertos, e las aguas dulces que y son, e las entradas, e las salidas, para guiar su nauio en saluo......La segunda, que sean esforçados para sofrir los peligros de la mar, e el miedo de los enemigos... ${ }^{60}$

Estos conocimientos son exactamente los mismos que se necesitan en el Atlántico, por lo que la ruta directa entre el litoral cantábrico y la zona de Saint-Mathieu no ofrecía ningún tipo de dificultades tanto geográficas como astronómicas. Los vientos dominantes en la zona comprendida entre la Península e Inglaterra son en su mayoría de componente oeste por lo que en caso de dificultades, los buques eran lanzados hacia las costas europeas. Por otra parte, ya hemos visto que el rumbo a seguir no ofrecia ningún tipo de dificultad pues se trataba de rumbo norte - desde Oviedoo noreste desde el cabo Ortegal. Es decir, es un rumbo en latitud y no en longitud, lo que facilita la navegación con instrumentos de sobra conocidos como el astrolabio ${ }^{61}$.

En 1169 se daba el tratado de Portovenere entre Génova y Pisa por el cual Génova imponía serios inconvenientes para la navegación pisana hacia las costas catalanas, lo que les obligaba a navegar por alta mar desde Noli a Salou. Orvietani Busch. S. Medieval Mediterranean ports..., p. 228.

:9amón LLULL. Arbre de Sciencia. Volumen I, De les fulles de larbre humanal.

6C Partida II, título XXIII, ley V.

"En la navegación los rumbos más problemáticos eran los que implicaban un desplazamiento en longitud, es decir hacia el Este o el Oeste, pues para su cálculo es necesario contar con instrumentos precisos que permitan saber el tiempo transcurrido y obtener de esta forma la distancia recorrida por el buque. Mientras que en la navegación en latitud -rumbo norte o sur-se puede obtener el avance del buque por la observación de los astros. 
Pero las navegaciones de naves mediterráneas en aguas atlánticas no se producian sin haber adaptado las naves para ese mar. En las atarazanas mallorquinas se conocían las características que debían tener los buques para poder navegar por el Atlántico. En 1328 el patrón mallorquín Guillem des Orts mandaba al mestre mayor Johannes Cucliure construir una tarida con la que navegar por el mediterráneo. Tres años más tarde decide cambiar de ámbito comercial y navegar hacia Salé en busca del preciado grano ante la carestía del mismo en Mallorca. Para ello contacta con el constructor de la tarida quien realizará en las atarazanas los cambios necesarios para adaptarla a la navegación atlántica: ....ad dictum viagum, scilicet, apud Sale fuit nouiter adaptata et bene preparata... ${ }^{62}$

\section{Instrumentos náuticos}

Pero la navegación no se limita a la estima sino que la geometría constituye un factor importante ya que ...la navegació naix i se deriva de la Geometria i de l'Aritmetica car la nau que a un temps se troba en un lloc, en altre temps està en un lloc diferent.. ${ }^{63}$

Para poder seguir estos rumbos los marinos contaban, ya desde la Antigüedad, con el cuadrante ${ }^{64}$ y el astrolabio ${ }^{65}$ para poder medir la latitud ${ }^{66}$ y comprobar de esta forma el avance del buque. Ramón Llull, al hablar de astrología, nos describe la utilidad del astrolabio para determinar la posición a través de las estrellas: ... Per aquelles -las constelaciones- fa hom iudicis de les furtunes saiús, enaxi con l'astralabri és nasasari astrument a conèxer l'asguardament et asituament dels corsas dasús et de lurs moviments ${ }^{67}$. Es decir, la observación de la altura de algunas estrellas - corsas dasús- de las cuales se conocia su declinación - lurs moviments-, permitía liegar a conocer la latitud del observador ${ }^{68}$. Estos

Archivo del Reino de Mallorca, Suplicacions 8, folio 33r.

Ramón LuUL, Ars Magna generalis et uitima, capitulo CXI.

$\rightarrow$ Ptolomeo ya hace reterencia al uso de los cuadrantes. Se cree que el cuadrante seria el primer instrumento introducido en la náutica. Los cuadrantes astrológicos presentaban una leyenda con los valores de las tangentes y cotangentes de ciertos ángulos. A partir del siglo XIII se completaba con una regla de cálculo para el uso astronómico, llamado cursor, que se apoyaba en la escala de alturas y podia correr a lo largo de ella. Alburqueroue, Luis de. Introduçao... p. 231.

"Desde finales del siglo VIII ya se conoce el tratado de Massala sobre el astrolabio y sus distintas traducciones que se fueron realizando a lo largo de la Edad Media como las de J. Hispanus o Rodolfo de Brujas. Por no hablar de la obra de Alfonso $X$ y sus Libros de Saber de Astronomia. En los diversos trabajos editados por VERNET bajo el título de Nuevos estudios sobre Astronomía española en el siglo de Alfonso $X$ se puede ver un completo estudio sobre el uso y funcionamiento de estos instrumentos.

.. Dado que la ruta directa hacia Flandes es básicamente dịrección norte -o sur de regreso-, con el cuadrante o ef astrolabio se podía saber en todo momenio el avance del buque gracias al cálculo de la altura del sol o las estrellas.

Ramón LLuLL. Novell tractac d'Astronomia - según un manuscrito del siglo XV del British Museum-

. Son muchos los tratados medievales sobre el astolabio donde se explica, siguiendo a Massala, esta sencilla operación: ... Si queremos tomar la latitud de un lugar durante la noche, tomaremos la altura de una estrella en el momento en que esté en el meridiano y esperaremos. Después buscaremos su declinación con respecto al ecuador y asi procederemos como hizimos con el sol. Millás Vallicrosa. Assaig d'Historia... p. 38. 
mismos conocimientos los refleja Alfonso $X$ en sus Libros del saber de Astrología y concretamente a través de los 135 capitulos de dedicados a Los libros del Astrolabio donde se estudia desde la construcción de dichos instrumentos hasta el cálculo de la latitud por ...la altura de cualquier estrella fija que non se asconda de yuso so la terra. Se complementan con las Tablas alfonsinas - calculadas con referencia al meridiano de Toledocomo elemento indispensable para poder obtener la latitud mediante la altura del sol y su declinación.

Pero las estrellas también tenían otros usos muy prácticos para los navegantes como pueden ser el conocer la hora de la noche, tal y como nos lo describe Cresques Abraham en su famoso Atlas. La observación de dos estrellas - llamadas por los navegantes Dos Hermanos- cercanas a una constelación situada al Norte y conocida por estos mismos navegantes como Carro - también conocida por los marinos italianos o mallorquines como Corn o Corno-, permite conocer la hora de la noche en cualquier época del año ${ }^{69}$. Años antes, Llull describía en su libro De Medicina un instrumento similar al que llama Astrolabi nocturni o Sphera nocturni con el cual se podia calcular la hora nocturna, mediante la observación de las estrellas, para poder administrar durante la noche los medicamentos a los enfermos.

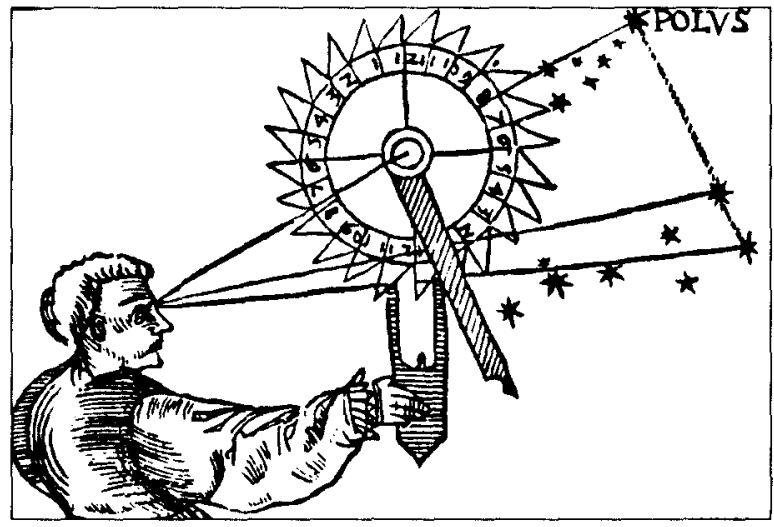

Uso del nocturnilabio

69 ...Devets saber encara que si es tems clar encara que si volets saber quants hores avets de la nit, que ho podets saber ab çerta raho e bona e breu. Devets saber que la tramuntana ha VIl astellas qui la vogen de nit e de jorn e aquestes han nom, sagons homens navegans, Charros. Enapres y ha dues astelles qui la vogen i son li pus pres e aquestes han nom Dos frares. Los quals II frares devets saber que migant dembre, que es la magor nit de lany, que aquels II frares, con lo sol es post, ques lunyen per tramuntana i fan lor cors tro al sol ixit per vent i dos quartes de vent, qui son ha XVI hores e mig. E migant març, que la nit e lo jorn son iguals, pugen per lavant e fan lor cors tro sol ixent e per XVI quartes de vent qui son XII hores. $E$ migant juny, que la nit es qurta de tot lany, pujen per migjorn e fan lor cors tro al sol ixit per $X$ quartes de vent qui son VII hore(s) e miga. E migant stembre, que son la nit el jorn cominals e aguals, se leven per ponent e fan lor cors tro al sol ixit per XVI quartes de vent qui son XII hores de nit $\mathrm{X} X \mid 1$ hores de jorn. $E$ ab aquesta rao podets saber quant avets de nit çertament ab que sia clar. Cresoues ABrAHAM. Atlas de 1375, lámina $1 \mathrm{~b}$. 
Pero se sigue preguntando Ramón Llull sobre las cuestiones de la mar y en el Arbre de Sciencia ${ }^{70}$ nos explica la forma en que los marinos calculaban la distancia recorrida por sus buques: ...Ramon, los mariners ¿com mesuren les milles en la mar? Los mariners consiren IIII vents generals, ço es saber levant, ponent, mitjorn e tramuntana; e consiren altres quatre vents qui ixen dels primers, ço es saber grec, exaloch, labeg e maestre. E consiren lo centre del cercle en lo qual los vents fan angles, a aprés consiren lo vent de levant anant la nau luny $C$ milles del centre, quantes milles ha tro lo vent d'exaloch e doblen les milles tro a CC milles que son CC del vent de levant tro lo vent d'exaloch per moltiplicament de les milles qui son del terme centenar de levant tro lo terme d'eixaloch. E de ço han instrument, carta $e$ compas, agulla e tramontana. En esta larga descripción realiza el beato un resumen de los conocimientos náuticos del momento. En primer lugar describe los 8 vientos principales ${ }^{71}$, que darán lugar a los 32 rumbos básicos, mediante los cuales -con un simple ejercicio de triangulaciónobtienen el avance efectivo del buque. Una explicación muy similar sobre este cálculo la da en el capítulo CXI de su Ars Magna cuando comenta que: ... suposant que en el punt en el qual venen a reunirse el quatre angles aguts sia la tramontana, o el port de la nau, i que la nau vol navegar vers Orient, anirà per Xaloc — sureste-i quant vagi per la quarta milla, aquestes quatre milles gairebé no compten per Xaloc, sinó per Llevant... i quant la nau camini vuit milles, en dirección a Xaloc, no compten sinó sis per Orient...

Ambas descripciones nos sitúan ante un antecedente del martologio como método de para calcular la posición en el mar, después de haber navegado en bolina, deduciendo los avances y retornos de la nave con respecto al rumbo deseado. Martologio que pronto irá acompañado de una tabla de cálculo para facilitar las operaciones y que se considera como el antecedente de los antiguos cartiers empleados durante siglos en la marina para calcular las distancias.

Pero sigue Llull mencionando otros tipos de instrumentos necesarios para la navegación de la época: ...E de ço han instrument, carta e compas, agulla e tramontana...

Las cartas náuticas se desarrollan como un elemento más de apoyo al marino en la navegación propia del momento, que es a la estima, y se forman como un producto artesanal de base totalmente empírica cuyo

${ }^{10}$ Arbre de Sciencia. Del Arbre questional, capítulo V: De les fuestions de les fulles del Arbre humanal / De les questions del habits, punto l: De les questions de geometria.

"Son los mismos que forman la rosa de los vientos de las cartas marinas del siglo XIII, en contraposición a la antigua rosa de 12 vientos que todavía vemos en obras del siglo XII como la Descriptio mappe mundi de Hugo de Saint-Victor, escrita hacia 1130: ...Primus itaque uentorum est subsolanus, secundus eurus, tertius euroauster. quartus auster, quintus austroafricanus, sextus africanus, septimus zephirus, octauus chorus, nonus circius, decimus septentrio, undecimus boreas, duodecimo uulturnus. Duo ex his tantum cardinales sunt auster, uidelicet, et septentrio. GAUTIER DALCHÉ, P. La Descriptio mappe mundi..., p. 134. 
origen lo podemos situar en los libros portulanos conocidos desde el siglo $X I$. No entro en las diversas opiniones sobre el origen de estas cartas pero si me gustaría destacar la existencia de tratados de geografía no sólo en Mediterráneo sino también en el mundo atlántico como el de Sa'id al Magrobí - escrito a mediados del siglo XIII- donde describe las costas atlánticas desde Marruecos hasta Vizcaya y haciendo constar los rumbos y distancias entre puertos, lo que implicaría el uso de cartas arrumbadas - de cuya existencia lamentablemente no tenemos constancia- también en la zona atlántica. En la zona mediterránea la primera carta conocida -la famosa Carta pisana-está fechada hacia 1290 , pero las referencias literarias se sitúan en 1270 cuando San Luis pide información al maestre de la nave -en medio de las muestras de desaprobación de otros maestres que consideraban que los vientos eran los propicios para llegar en 4 dias desde Aigües-Mortes hasta Cagliari- sobre su situación después de un temporal: ...pource que il lor sambloit que il métoient trop longuement à arriver au port de Chastiau-Castre en Sardine, où li Baron de cet pélerinage devoient arriver e attendre li uns l'autre. Pour ceste chose furent li maistre des nez mandé devant le roy, liquelz li demanda conbien il avoit d'espasse dou lieu où il estoient, jusques à Chastiau-Castre. Li maronnier respondirent au Roy sous parole doutable, e distrent que il creoient qu'il fussent près de terre, e moult se merveilloient qu'il tardoient tant qu'il ne le véoient. Lors firent apporter la mapemonde devant le Roy, e li mostrèrent le siége dou port de Chastiau-Castre e conbien il estoient près dou rivage... ...Endementres grans murmures e grans soupeçons mût encontre euls -los maestres de la nave -; car chascun disoit que le vent que il avoient eu après ce que il se partirent dou port d'Aigremorte, estoit soufisans e convenables pour mener les nez en quatre jours au port de Chastiau-Castre...

En cuanto al compás mencionado por Llull, no existe unanimidad en cuanto a su definición pues mientras algunos autores como Fernandez Navarrete lo identifican con el compás por todos conocido, otros autores lo identifican con los portulanos.

Ramón Llull siente una cierta fascinación por la aguja magnética y sus propiedades ...Car no es, Senyer, null home qui pogués apercebre ni saber tota la proprietat ni la conveniencia que la caramida e la agulla an en natura com la agulla se gir en la dressera de la tremuntana per lo tocament que fa en la caramida. ${ }^{72}$ La referencia más antiguas ${ }^{73}$ sobre el uso de la aguja, entre los europeos, la encontramos a fines del siglo XII o inicios del XIII en autores como Neckam o en la poesía de Guiot de Provins -escrita hacia 1205-donde explica que los mareantes la emplean en caso de mal tiempo:

Llibre de Contemplació en Deu, capitulo 171: Com hom aperceb e enten lo cors de la natura e les obres de natura.

No entro a analizar los posibles orígenes asiáticos o europeos de la aguja pues no es este el objeto del trabajo. 
...laide el bruinière... torne la pointe toute contre l'estoile quand la mer est obscure et brune... De la misma opinión es Neckam cuando comenta que la usan los marinos cuando éstos ...ignorant in quem mundi cardinem propra tendant. Es decir, el empleo de la aguja imantada se limitaba en sus orígenes a su uso cuando las circunstancias metereológicas impedian utilizar otros medios como el cálculo solar o el astronómico pues es bien sabido que el mareante ...sendressa en son viatge per les ensenyes de la estela e de les terres... y concretamente, tal y como hemos visto con el cuadrante y el astrolabio, ...los mariners con van per la mar, ques guien per la tremuntana... ${ }^{74}$

\section{CONCLUSIONES}

A lo largo del presente trabajo hemos visto a las naves del Norte europeo navegar en el Mediterráneo. La suma de los buques que llegaron a la cuenca mediterránea, en viajes directos desde Bretaña a Gijón, entre 1147 y 1217 asciende a algo más de 500 unidades sólo en las 4 expediciones de cruzados consultadas para el presente trabajo. Esta entrada de buques atlánticos no podía pasar desapercibida para los marinos mediterráneos que sin duda tomarian buena nota sobre las principales características técnicas de esos buques, adoptando las que consideraran útiles para sus necesidades. $^{75}$

Vemos pues un gran intercambio - profesional y técnico- entre ambos mundos antes de que los marinos del Mediterráneo se lanzaran a las aguas atlánticas con unas galeras adaptadas según el modelo oceánico y que podrán navegar sin problemas por unas rutas muy bien conocidas.

Para la navegación directa entre la costa gallega y el Canal de la Mancha los mareantes mediterráneos habian adquirido los conocimientos y los medios técnicos necesarios para realizar estas travesías, lo que redundaba en los mercaderes que, por su parte, se beneficiaban de la disminución de tiempo y costes. Esta ruta directa se vio rápidamente complementada con la presencia mediterránea en la ruta de cabotaje al menos desde el primer tercio del siglo XIV.

- Llibre de Contemplació en Deu, capítulos 280 y 117 respectivamente.

Pero no sólo conocian los diseños de los buques atlánticos por las entradas de éstos en el Mediterráneo, sino que a petición de Eduardo I son varios los genoveses que le construyen en la isla naves con vistas a una nueva cruzada. En 1274 Simone Mallone recibia el pago por una nave que le había construido y lo mismo ocurre con Bartolino Dentuto, quien también construye dos naves para el monarca. LoPEz.

Majorcans and Genoese... p. 1171. 


\section{BIBLIOGRAFÍA}

Alburquerque, Luis de. Introduçao à História dos descobrimentos portugueses. Publicaçōes Europa-America.

AL-IDRISI. Los caminos de al-Andalus en el siglo XII. Según "uns al-muhay wa-rawd al-furay" (solaz de corazones y prados de contemplación). Estudio, edición, traducción y anotaciones de ABID MIZAL, Jasim. CSIC. Instituto de Filología. Madrid, 1989.

BAstaRd de PERE, René. Navires méditerranéens du temps de Saint Louis. Revue d'Histoire Économique et Sociale.

Cántigas de Santa Maria. Editadas por Walter METTMANN. 2 tomos. Edicións Xerais de Galicia S.A. Vigo, 1981.

Capmany y de Monpalau, Antonio. Memorias históricas sobre la Marina, comercio y artes de la antigua ciudad de Barcelona. 3 Vols. Cámara Oficial de Comercio y Navegación de Barcelona. 1961.

Chaunu, Pierre. La expansión europea (siglos XIII al XV). Editorial Labor. Barcelona, 1972.

Crónica de Alfonso X. Edición de Manuel González Jiménez. Real Academia Alfonso X el Sabio, 1998.

DARSERL, J. La vie maritime sur les côtes du Léon sous l'Ancien Régime. Société Archéologique du Finistère.

DESCOBRIMENTOS PORTUGUESES. Silva Marques, Joao Martins. Ediçao do Instituto para a Alta Cultura. Lisboa, 1944.

Doemaero, Renée. Les Galères génoises dans la Manche et la Mer du Nord à la fin du XIII et au début du XIV s. Bulletin de l'Institut Historique Belge de Rome. Bruxelles-Rome, 1938.

EL LIBRO DE LA "REGLA COLORADA"DE LA CATEDRAL DE OVIEDO. Estudio y edición. Rodríguez Diaz, Elena. Real Instituto de Estudios Asturianos. Oviedo, 1995

Ferreira Priegue, Elisa. Galicia en el comercio marítimo medieval. Fundación «Pedro Barrie de la Maza». La Coruña, 1988.

Ferreiro Alemparte, Jaime. Arribadas de Normandos y Cruzados a las costas de la Península Ibérica. Sociedad española de Estudios Medievales. Madrid, 1999.

Gautier Dalché, Patrick.

CARTE MARINE ET PORTULAN AU XIJe SIECCLE. Le "Liber de existencia riveriarum et forma maris nostri mediterranei" (Pise, circa 1200). École française de Rome. Palais Farnèse. 1995. LA "DESCRIPTIO MAPPE MUNDI"DE HUGUES DE SAINT-VICTOR. Texte inédit avec introduction et commentaire. Études Augustiennes. Paris, 1988.

GonzALEZ, Julio. Repartimiento de Sevilla. CSIC. Escuela de Estudios Medievales. Madrid, 1951.

GOYHENECHE, E. Bayonne et la región bayonnaise du Xlle au XIVe siècle. Études d'histoire économique et sociale. Universidad del Pais Vasco, 1990.

HISTOIRE DE SAINT LOUIS par sire de Joinville; LES ANNALES DE SON REGNE par Guillaume de Nancis; SA VIE ET SES MIRACLES par le Confèsseur de la Reine Margueritte. Le tout publié d'après les Manuscrits de la Bibliothèque du Roi et accompangé d'un Glossaire. A Paris, de I'Imprimerie Royale. M.DCC.XI.

LAS SIETE PARTIDAS. Glosadas por el Licenciado Gregorio Lopez. Editadas por el Boletín Oficial del Estado. Madrid, 1985.

López, Roberto S. Majorcans ad Genoese on the North Sea route in the thirteenth century. Revue Belge de Philologie et d'Histoire. Tome XXIX (1951), n. ${ }^{\circ}$. Bruxelles, 1951.

Luul, Ramón.

Libre de Contemplació en Deu. 3 volúmenes. M. Obrador Bennassar. Comissió editora Lulliana. Palma de Mallorca, 1906.

Arbre de Sciencia. 7 volúmenes. Mossén Salvador Galmés. Comissió editora Lulliana. Palma de Mallorca, 1917.

Madufell Marinón, Josep Maria. Ordenanzas maritimas de 1331 y 1333. A.H.D.E., tomo XXXI. Madrid, 1961.

MAPAMUNDI DEL AÑO 1375 de CRESQUES ABRAHAM y JAFUDA CRESQUES. EBRISA, S.A. Barcelona, 1983. 
Millas Vallicrosa. Assaig d'Història de les Idees Fisiques e Matematiques. Barcelona, 1931.

Mollat DU JoRoIn, Michel. Europa y el mar. Editorial Crítica. Barcelona, 1993.

Murino, Mario. Andar per mare nel Medioevo. Le antiche consuetudini marittime italiane. Vecchio Faggio Editore. Chieti, 1988

OrviETANI Busch, Silvia. Medieval Mediterranean ports. The Gatalan and Tuscan Coasts, 1100 to 1235. Leiden; Boston; Köln; Brill, 2001. (The medieval Mediterranean, vol. 32).

PETTI BALBI, Giovanna. Mercanti e "nationes» nelle Fiandre: i genovesi in età bassomedievale. Gisem-Edizioni ETS. Pisa

PICAD, Christophe. L'océan Atlantique musulman. De la conquête arabe à l'époque almohade. Navigation et mise en valeur des côtes d'al-Andalus et du Maghreb occidental (PortugalEspagne-Maroc). Éditions Maisonneuve \& Larose / Éditions UNESCO. Paris, 1997

PISTARINo, Geo. La capitale del Mediterraneo: Génova nel medioevo. Instituto Internacionale di Studi Liguri. Bordighera, 1993

PORTUGALIAE MONUMENTA HISTORICA, a saeculo octavo post Christum usque ad quintumdecimum. Scriptores, volumen I. Reedición de KRAUS REPRINT LTD. Nendeln, Liechtenstein, 1967.

RenouARD, Y. Les hommes d'affaires italiens à La Rochelle au Moyen Âge. En "Études d'Histoire Médiévale". S.E.V.P.E.N. París, 1968.

Sevillano Colom, Fco. De Venecia a Flandes (Vía Mallorca y Portugal, siglo XIV). B.S.A.L. Paima de Mallorca, 1968.

SotTAS, J. Les messageries maritimes de Venise au XV siècle. París, 1936.

ToRres Fontes, Juan. La Orden de Santa Maria de España. Miscelánea Medieval Murciana, vol. III. Universidad de Murcia, 1977.

TOUCHARD, Henri. Le commerce maritime breton à la fin du Moyen Âge. Annales Litèraires de L'Université de Nantes 1. Collection "Les belles lettres". París, 1967.

VERNET, Juan. Nuevos estudios sobre Astronomia española en el siglo de Alfonso $X$. CSIC. Institución "Milá i Fontanals". Barcelona, 1983. 